\section{Reply to Bellíssimo-Rodrigues et al.}

TO THE EDITOR-In the intensive care unit at my institution, surveillance cultures are used to guide the choice of empirical antibiotic therapy. ${ }^{1}$ This strategy is an alternative to an approach in which ultra-broad-spectrum antibiotics are initially used empirically, after which (if possible) an agent with a narrower spectrum is administered once the causative microorganism and its antibiogram are known. ${ }^{2}$ In the June 2005 issue of the journal, my colleagues and I reported our long-term experience with the use of routine surveillance cultures in an intensive care unit by means of a study designed to measure the value of this strategy in terms of the appropriateness of antibiotic therapy. ${ }^{3}$

Bellíssimo-Rodrigues et al. ${ }^{4}$ question the accuracy of our analyses. First, they assume that a potential bias exists with respect to the group of patients without prior colonization, because it may have included patients who had been colonized by an antibiotic-resistant gram-negative bacterium different from the one subsequently recovered from the blood. Indeed, it seems logical that, in such situations, the empirical regimen would be effective against the colonizing bacterium and, therefore, increase the likelihood that appropriate antibiotic therapy would be administered in the event that the blood culture yielded another resistant microorganism. Thus, the rate of appropriate antibiotic therapy might be falsely high in this particular group. However, exclusion of these patients would only strengthen the results, because the difference in the rate of appropriate antibiotic therapy between patients with versus patients without prior colonization was already statistically significant. Therefore, the potential bias suggested by Bellíssimo-Rodrigues et al. ${ }^{4}$ could not alter the final results.

A second concern raised by Bellíssimo-Rodrigues et al. ${ }^{4}$ is the presence of producers of extended-spectrum $\beta$-lactamases, which is indeed problematic with respect to judging the appropriateness of antibiotic therapy. During the study period, the susceptibility patterns of extended-spectrum $\beta$ lactamase-producing isolates were not altered, as determined on the basis of the most-recent recommendations of the Clinical and Laboratory Standards Institute (formerly known as the NCCLS). However, as indicated in the article, at my institution ceftazidime resistance is a marker for extended-spectrum $\beta$-lactamase producers. When a ceftazidime-resistant isolate is recovered, a warning is added to the microbiology report stating that the patient is colonized or infected with an extended-spectrum $\beta$-lactamase-producing bacterium. In this way, possible misinterpretation of the appropriateness of therapy can be avoided, although it remains a point of discussion in cases in which the empirical treatment consists of antibiotics associated with $\beta$-lactamase inhibitors (including piperacillin and tazobactam). In any case, I recognize that the concern raised by Bellíssimo-Rodrigues et al. ${ }^{4}$ is pertinent in the sense that the definition of appropriate antibiotic therapy given in the article was incomplete and did not represent the true circumstances in which the results were obtained. Appropriate therapy should have been defined as a regimen that contains an antibiotic with in vitro and clinical activity against the causative pathogen, which is the definition my colleagues and I had used previously.

Stijn Blot, $\mathrm{PhD}$

Prof. Blot is from the Intensive Care Department, Ghent University Hospital, Ghent, Belgium.

Infect Control Hosp Epidemiol 2006; 27:105

(C) 2006 by The Society for Healthcare Epidemiology of America. All rights reserved. 0195-9417/2006/2701-0028\$15.00.

\section{REFERENCES}

1. Blot S, Vandewoude K. Early detection of systemic infection. Acta Clin Belg 2004; 59:20-23.

2. Colardyn F. Appropriate and timely empirical antimicrobial treatment of ICU infections-a role for carbapenems. Acta Clin Belg 2005; 60:51-62.

3. Blot S, Depuydt P, Vogelaers D, et al. Colonization status and appropriate antibiotic therapy for nosocomial bacteremia caused by antibiotic-resistant gram-negative bacteria in an intensive care unit. Infect Control Hosp Epidemiol 2005; 26:575-579.

4. Bellissimo-Rodrigues F, de Castro Figueiredo JF, Martinez R. Colonization by antibiotic-resistant gram-negative bacteria and appropriate empirical antibiotic therapy in intensive care unit patients [letter]. Infect Control Hosp Epidemiol 2006 27:104 (in this issue).

5. Blot SI, Depuydt P, Annemans L, et al. Clinical and economic outcomes in critically ill patients with nosocomial catheter-related bloodstream infections. Clin Infect Dis 2005; 41:1591-1598.

6. Myny D, Depuydt P, Colardyn F, Blot S. Ventilator-associated pneumonia in a tertiary care ICU: analysis of risk factors for acquisition and mortality. Acta Clin Belg 2005; 60:114-121. 